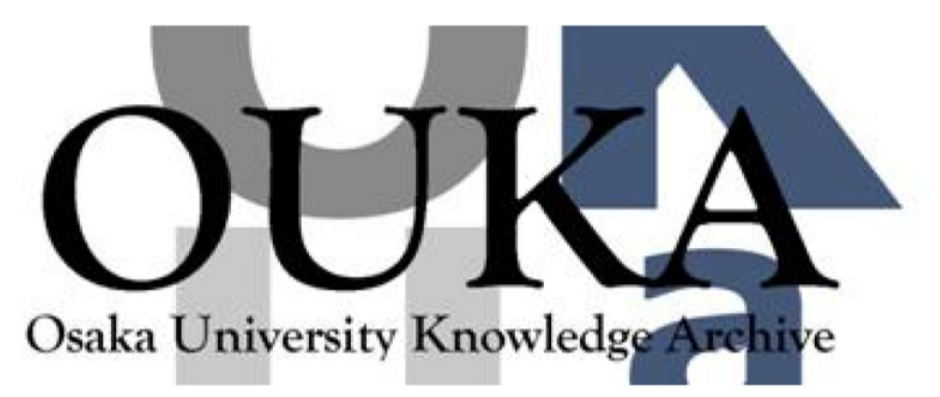

\begin{tabular}{|c|c|}
\hline Title & $\begin{array}{l}\text { Spin pumping from finite-sized electron systems } \\
\text { in ballistic and diffusive transport regimes }\end{array}$ \\
\hline Author (s) & Hattori, Kiminori \\
\hline Citation & PHYSICAL REVIEW B. 75(20) p. 205302-1-p. 205302-8 \\
\hline Issue Date & $2007-05-02$ \\
\hline oaire:version & VoR \\
\hline URL & https://hdl. handle. net/11094/3113 \\
\hline rights & $\begin{array}{l}\text { Hattori, Kiminori, Physical Review B, 75, 20, } \\
205302 \text {, 2007-05-02. "Copyright } 2007 \text { by the } \\
\text { American Physical Society." }\end{array}$ \\
\hline Note & \\
\hline
\end{tabular}

Osaka University Knowledge Archive : OUKA

https://ir. Library. osaka-u. ac. jp/

0saka University 


\title{
Spin pumping from finite-sized electron systems in ballistic and diffusive transport regimes
}

\author{
Kiminori Hattori \\ Department of Systems Innovation, Graduate School of Engineering Science, Osaka University, Toyonaka, Osaka 560-8531, Japan
}

(Received 18 December 2006; published 2 May 2007)

\begin{abstract}
This paper presents a theory for spin pumping from finite-sized conductors at paramagnetic resonance. The spin generation and transport in the system exposed to a rotating magnetic field are analyzed by using the nonequilibrium Green's-function formalism, showing that a time-independent spin current is produced with no charge current when the electrochemical potentials in all leads attached to the system are identical. A physically transparent formulation for the spin pumping is derived in terms of the spin-current continuity equation with the source term due to the rotating field. The characteristics of spin-pumping systems are investigated by numerical calculation. In the ballistic transport regime, the pumped spin at resonance is augmented normally with the system size and the pumping intensity, insofar as the system contacts ideal leads that transport spin to the outside world and the pumping field is perturbatively weak. In the diffusive transport regime, the spin pumping is strongly enhanced with the degree of static disorder. The mechanism of enhancement is discussed in view of the weak localization.
\end{abstract}

DOI: 10.1103/PhysRevB.75.205302

PACS number(s): 72.25.Dc, 72.10.Bg, 73.23.-b, 71.70.Ej

\section{INTRODUCTION}

The emerging field of spintronics, which aims to manipulate electron spin in addition to charge in solid-state systems, has attracted much recent interest. One central issue in this field is how to efficiently generate spin current in nonmagnetic metals or semiconductors. Proposals for generating spin current include spin injection from ferromagnetic metals or magnetic semiconductors, ${ }^{1-4}$ a spin battery consisting of a ferromagnet at the ferromagnetic resonance contacted by a normal metal or a doped semiconductor, ${ }^{5}$ a quantum-dotbased spin pump that uses the combination of a static magnetic field and an oscillating electric field, ${ }^{6-8}$ and spin Hall effects exploiting the intrinsic spin-orbit coupling in nonmagnetic semiconductors. ${ }^{9,10}$ Alternatively, it has been suggested that a quantum dot at paramagnetic resonance provides a generating source for spin current. ${ }^{11-13}$ In this spinpumping device, the spin-degenerate electron levels in the dot are split by a static longitudinal magnetic field. Applying a weak transverse magnetic field oscillating or rotating at the resonant frequency couples the two Zeeman levels and makes a spin-flip transition between them. If the electrochemical potential is suitably positioned between the two spin levels, a spin- $\uparrow$ (spin- $\downarrow$ ) electron created by spin-flip transition is ejected into an infinitely extended reservoir in contact with the dot, leaving a hole which is subsequently filled with a spin- $\downarrow$ (spin- $\uparrow$ ) electron injected from the reservoir. This process repeats to establish a continuous outgoing (ingoing) flow of spin current. The spin current is reversed by reversing the longitudinal field and the rotation of transverse field.

In this paper, we consider the spin pumping from finitesized systems at paramagnetic resonance. The continuity equation for spin current, the microscopic details of which are well defined in the finite-sized system, provides a physically transparent formulation for the spin pumping, in combination with the theoretical analysis based on the nonequilibrium Green's-function formalism. ${ }^{14-17}$ The characteristics of spin-pumping systems are investigated by numerical cal- culations for one- and two-dimensional models. The calculation shows that the pumped spin varies significantly with the dimensionality, the system size, the intensity of rotating field, the detuning from resonance, and the static disorder. In the ballistic transport regime, a simple analytical expression that predicts the pumped spin is deduced by examining the numerical results. An interesting observation in the diffusive transport regime is that the spin pumping is remarkably enhanced with increasing static disorder. The mechanism of enhancement is discussed in terms of the weak localization.

\section{THEORETICAL ANALYSIS AND FORMULATION}

Throughout this paper, we shall work in units where $\hbar$ $=e=1$. We begin by considering a noninteracting electron gas exposed to a rotating transverse magnetic field $\widetilde{\mathbf{B}}(t)$ $=\widetilde{B}\left(\mathbf{e}_{x} \cos \omega t+\mathbf{e}_{y} \sin \omega t\right)$ in addition to a static longitudinal field $\overline{\mathbf{B}}=\bar{B} \mathbf{e}_{z}$, with $\mathbf{e}_{x, y, z}$ being the unit vectors in Cartesian coordinates. The Zeeman interactions under these external fields are described by $\bar{H}=\bar{\omega} S_{z}$ and $\tilde{H}(t)$ $=(\widetilde{\omega} / 2) \Sigma_{ \pm} S_{ \pm} \exp (\mp i \omega t)$, where $\bar{\omega}=\gamma \bar{B}$ and $\widetilde{\omega}=\gamma \widetilde{B}$ are the Larmor frequencies, $\gamma(>0)$ is the gyromagnetic ratio, and $S_{z}$ and $S_{ \pm}=S_{x} \pm i S_{y}$ are the spin operators. Including the kineticenergy part $H_{0}=-(1 / 2 m) \nabla^{2}$ with the electron mass $m$, the total Hamiltonian can be written as $H(t)=H_{0}+\bar{H}+\tilde{H}(t)$. This expression neglects the orbital effects and simplifies the theoretical analysis of spin pumping. ${ }^{18}$

The Schrödinger equation $i \partial \psi(\mathbf{r}, t) / \partial t=H(t) \psi(\mathbf{r}, t)$ for the fermion field operator $\psi(\mathbf{r}, t)$ leads to the standard sourceless continuity equation

$$
\frac{\partial}{\partial t} \rho_{\text {charge }}(\mathbf{r}, t)+\nabla \cdot \mathbf{j}_{\text {charge }}(\mathbf{r}, t)=0
$$

between the charge density operator $\rho_{\text {charge }}(\mathbf{r}, t)$ $=\psi^{\dagger}(\mathbf{r}, t) \psi(\mathbf{r}, t)$ and the charge-current-density operator $\mathbf{j}_{\text {charge }}(\mathbf{r}, t)=\operatorname{Re} \psi^{\dagger}(\mathbf{r}, t) \mathbf{v} \psi(\mathbf{r}, t)$, while the spin-density opera- 
tor $\rho_{\text {spin }}(\mathbf{r}, t)=\psi^{\dagger}(\mathbf{r}, t) S_{z} \psi(\mathbf{r}, t)$ and the spin-current-density operator $\mathbf{j}_{\text {spin }}(\mathbf{r}, t)=\operatorname{Re} \psi^{\dagger}(\mathbf{r}, t) S_{z} \mathbf{v} \psi(\mathbf{r}, t)$ obey the continuity equation

$$
\frac{\partial}{\partial t} \rho_{\text {spin }}(\mathbf{r}, t)+\nabla \cdot \mathbf{j}_{\text {spin }}(\mathbf{r}, t)=g_{\text {spin }}(\mathbf{r}, t)
$$

containing the source term represented as $g_{\text {spin }}(\mathbf{r}, t)$ $=\widetilde{\omega} \operatorname{Im} \psi^{\dagger}(\mathbf{r}, t) S_{+} \psi(\mathbf{r}, t) \exp (-i \omega t)$, where $\quad \mathbf{v}=-(i / m) \nabla$ denotes the kinetic velocity, and $\operatorname{Re} A$ and $\operatorname{Im} A$ are defined as $\left(A+A^{\dagger}\right) / 2$ and $\left(A-A^{\dagger}\right) / 2 i$, respectively. The operator $g_{\text {spin }}(\mathbf{r}, t)$, which we call the spin generation rate, can be reexpressed as $\mathbf{e}_{z} \cdot\left[\gamma \widetilde{\mathbf{B}}(t) \times \psi^{\dagger}(\mathbf{r}, t) \mathbf{S} \psi(\mathbf{r}, t)\right]$ and corresponds to the out-of-plane projection of spin torque density due to the rotating field on the $x y$ plane. In what follows, we will show, through an analysis based on the nonequilibrium Green'sfunction formalism, that the physics of spin pumping is understood basically in terms of the spin-current continuity [Eq. (2)].

A spin-pumping system, which can be $d=1,2$, or 3 dimensional, is modeled by considering a finite-sized conductor sample in contact with ideal leads that are maintained at individual electrochemical potentials without exposure to the rotating field. In the tight-binding representation on a $d$-dimensional lattice with lattice spacing $a$, the Hamiltonian describing the sample region, $H(t)=H_{0}+\bar{H}+\widetilde{H}(t)$, is composed of

$$
\begin{gathered}
H_{0}=-t_{0} \sum_{\mathbf{r}, \sigma \pm \mathbf{a}} \sum_{\mathbf{r} \sigma} c_{\mathbf{r} \pm \mathbf{a} \sigma}^{\dagger}+2 d t_{0} \sum_{\mathbf{r}, \sigma} c_{\mathbf{r} \sigma}^{\dagger} c_{\mathbf{r} \sigma}, \\
\bar{H}=\frac{1}{2} \bar{\omega} \sum_{\mathbf{r}, \sigma} \sigma c_{\mathbf{r} \sigma}^{\dagger} c_{\mathbf{r} \sigma}, \\
\widetilde{H}(t)=\frac{1}{2} \widetilde{\omega} \sum_{\mathbf{r}, \sigma} c_{\mathbf{r} \sigma}^{\dagger} c_{\mathbf{r},-\sigma} \exp (-i \sigma \omega t) .
\end{gathered}
$$

In these expressions, $c_{\mathbf{r} \sigma}\left(c_{\mathbf{r} \sigma}^{\dagger}\right)$ is the annihilation (creation) operator of an electron at position $\mathbf{r}$ with spin $\sigma(= \pm 1$ for spin- $\uparrow$ and $\downarrow$ states, respectively), $t_{0}=1 / 2 m a^{2}$ is the hopping energy, and $\mathbf{a}$ is the unit lattice vector.

Two nonequilibrium one-particle propagators, the lesser Green's function $G_{\sigma \sigma^{\prime}}^{<}\left(t, t^{\prime}\right)$, and the retarded Green's function $G_{\sigma \sigma^{\prime}}^{R}\left(t, t^{\prime}\right),{ }^{14-17}$ are defined by

$$
\begin{gathered}
{\left[G_{\sigma \sigma^{\prime}}^{<}\left(t, t^{\prime}\right)\right]_{\mathbf{r r}^{\prime}}=i\left\langle c_{\mathbf{r}^{\prime} \sigma^{\prime}}^{\dagger}\left(t^{\prime}\right) c_{\mathbf{r} \sigma}(t)\right\rangle,} \\
{\left[G_{\sigma \sigma^{\prime}}^{R}\left(t, t^{\prime}\right)\right]_{\mathbf{r r}^{\prime}}=-i\left\langle\left\{c_{\mathbf{r} \sigma}(t), c_{\mathbf{r}^{\prime} \sigma^{\prime}}^{\dagger}\left(t^{\prime}\right)\right\}\right\rangle \theta\left(t-t^{\prime}\right) .}
\end{gathered}
$$

The retarded Green's function obeys the following equation of motion:

$$
\begin{gathered}
i \frac{\partial}{\partial t} G_{\sigma \sigma^{\prime}}^{R}\left(t, t^{\prime}\right)-\sum_{\sigma^{\prime \prime}} H_{\sigma \sigma^{\prime \prime}}(t) G_{\sigma^{\prime \prime} \sigma^{\prime}}^{R}\left(t, t^{\prime}\right) \\
-\int_{-\infty}^{\infty} d t^{\prime \prime} \sum_{\sigma}^{R}\left(t-t^{\prime \prime}\right) G_{\sigma \sigma^{\prime}}^{R}\left(t^{\prime \prime}, t^{\prime}\right) \\
=\delta_{\sigma \sigma^{\prime}} \delta\left(t-t^{\prime}\right),
\end{gathered}
$$

with $\Sigma_{\sigma}^{R}(t)=\Sigma_{\alpha} \Sigma_{\alpha \sigma}^{R}(t)$, where $\Sigma_{\alpha \sigma}^{R}(t)=V_{\alpha} g_{\alpha \sigma}^{R}(t) V_{\alpha}^{\dagger}$ is the retarded self-energy due to lead $\alpha, V_{\alpha}$ is the hopping matrix connecting lead $\alpha$ with the sample, and $g_{\alpha \sigma}^{R}(t)$ is the retarded Green's function of the isolated semi-infinite lead. The equation of motion is solved by applying the double-time Fourier transformation. The solution is formulated as

$$
G_{\sigma \sigma^{\prime}}^{R}\left(t, t^{\prime}\right)=R_{\sigma \sigma^{\prime}}^{R}\left(t-t^{\prime}\right) \exp (-i \sigma \omega t / 2) \exp \left(i \sigma^{\prime} \omega t^{\prime} / 2\right),
$$

where $R_{\sigma \sigma^{\prime}}^{R}\left(t-t^{\prime}\right)$ depending only on the time difference $t$ $-t^{\prime}$ corresponds to a retarded Green's function in a reference frame rotating at frequency $\omega$, in which the magnetic field on the $x y$ plane becomes static. The transformation to the rotating frame is defined by a unitary operator $U(t)$ $=\exp \left[(i \omega t / 2) \Sigma_{\mathbf{r}, \sigma} \sigma c_{\mathbf{r} \sigma}^{\dagger} c_{\mathbf{r} \sigma}\right]$. The Fourier transforms of $R_{\sigma \sigma^{\prime}}^{R}(t)$ are expressed as

$$
\begin{gathered}
R_{\sigma \sigma}^{R}(\varepsilon)=\widetilde{G}_{\sigma}^{R}(\varepsilon), \\
R_{\sigma,-\sigma}^{R}(\varepsilon)=\bar{G}_{\sigma}^{R}(\varepsilon) \widetilde{\Omega} \widetilde{G}_{-\sigma}^{R}(\varepsilon),
\end{gathered}
$$

with $\quad \bar{G}_{\sigma}^{R}(\varepsilon)=G_{\sigma}^{R}(\varepsilon+\sigma \omega / 2), \quad \widetilde{G}_{\sigma}^{R}(\varepsilon)=\left\{\left[\bar{G}_{\sigma}^{R}(\varepsilon)\right]^{-1}-\widetilde{\Sigma}_{\sigma}^{R}(\varepsilon)\right\}^{-1}$, $\widetilde{\Sigma}_{\sigma}^{R}(\varepsilon)=\widetilde{\Omega} \bar{G}_{-\sigma}^{R}(\varepsilon) \widetilde{\Omega}$, and $\widetilde{\Omega}_{\mathrm{rr}^{\prime}}=(\widetilde{\omega} / 2) \delta_{\mathrm{rr}^{\prime}}$. Here, $G_{\sigma}^{R}(\varepsilon)=[\varepsilon$ $\left.-H_{\sigma}-\Sigma_{\sigma}^{R}(\varepsilon)\right]^{-1}$ represents the retarded Green's function of the spin- $\sigma$ subsystem in the laboratory frame when the rotating field is absent. Note that the diagonal part of Hamiltonian in spin space, $H_{\sigma}=[H(t)]_{\sigma \sigma}$, is time independent. The contribution due to the uniform rotating field with the Larmor frequency $\widetilde{\omega}$ is contained in the diagonal matrix $\widetilde{\Omega}$, and hence $\widetilde{\Sigma}_{\sigma}^{R}(\varepsilon)$ is regarded as the retarded self-energy due to the rotating field. The present formulation is applicable to a nonuniform rotating field with $\widetilde{\omega}(\mathbf{r})$ for which $\widetilde{\Omega}_{\mathbf{r r}^{\prime}}$ $=(1 / 2) \widetilde{\omega}(\mathbf{r}) \delta_{\mathbf{r r}^{\prime}}$. In the adiabatic limit, the energy shift $\sigma \omega / 2$ in the rotating frame is negligible so that $\bar{G}_{\sigma}^{R}(\varepsilon)=G_{\sigma}^{R}(\varepsilon)$.

The lesser Green's function satisfies the following Keldysh equation:

$$
\begin{aligned}
G_{\sigma \sigma^{\prime}}^{<}\left(t, t^{\prime}\right)= & \sum_{\sigma^{\prime \prime}} \int_{-\infty}^{\infty} d t_{1} \int_{-\infty}^{\infty} d t_{2} G_{\sigma \sigma^{\prime \prime}}^{R}\left(t, t_{1}\right) \\
& \times \Sigma_{\sigma^{\prime \prime}}^{<}\left(t_{1}-t_{2}\right) G_{\sigma^{\prime \prime} \sigma^{\prime}}^{A}\left(t_{2}, t^{\prime}\right),
\end{aligned}
$$

with $G_{\sigma \sigma^{\prime}}^{A}\left(t, t^{\prime}\right)=\left[G_{\sigma^{\prime} \sigma}^{R}\left(t^{\prime}, t\right)\right]^{\dagger}$ and $\Sigma_{\sigma}^{<}(t)=\Sigma_{\alpha} \Sigma_{\alpha \sigma}^{<}(t)$, where $\Sigma_{\alpha \sigma}^{<}(t)=V_{\alpha} g_{\alpha \sigma}^{<}(t) V_{\alpha}^{\dagger}$ is the lesser self-energy due to lead $\alpha$ and $g_{\alpha \sigma}^{<}(t)$ is the lesser Green's function of the isolated semiinfinite lead. The lesser self-energy is Fourier transformed into $\Sigma_{\alpha \sigma}^{<}(\varepsilon)=i f_{\alpha}(\varepsilon) \Gamma_{\alpha \sigma}(\varepsilon)$, where $\Gamma_{\alpha \sigma}(\varepsilon)=-2 \operatorname{Im} \Sigma_{\alpha \sigma}^{R}(\varepsilon)$ and $f_{\alpha}(\varepsilon)$ is the Fermi-Dirac distribution function in lead $\alpha$. Performing the integration over $t_{1}$ and $t_{2}$, we obtain

$$
G_{\sigma \sigma^{\prime}}^{<}\left(t, t^{\prime}\right)=R_{\sigma \sigma^{\prime}}^{<}\left(t-t^{\prime}\right) \exp (-i \sigma \omega t / 2) \exp \left(i \sigma^{\prime} \omega t^{\prime} / 2\right) .
$$

The lesser Green's function $R_{\sigma \sigma^{\prime}}^{<}(t)$ in the rotating frame is expressed in Fourier space as 


$$
R_{\sigma \sigma^{\prime}}^{<}(\varepsilon)=\sum_{\sigma^{\prime \prime}} R_{\sigma \sigma^{\prime \prime}}^{R}(\varepsilon) \bar{\Sigma}_{\sigma^{\prime \prime}}^{<}(\varepsilon) R_{\sigma^{\prime \prime} \sigma^{\prime}}^{A}(\varepsilon),
$$

where $R_{\sigma \sigma^{\prime}}^{A}(\varepsilon)=\left[R_{\sigma^{\prime} \sigma}^{R}(\varepsilon)\right]^{\dagger}, \bar{\Sigma}_{\sigma}^{<}(\varepsilon)=i \Sigma_{\alpha} f_{\alpha \sigma}(\varepsilon) \bar{\Gamma}_{\alpha \sigma}(\varepsilon), f_{\alpha \sigma}(\varepsilon)$ $=f_{\alpha}(\varepsilon+\sigma \omega / 2)$, and $\bar{\Gamma}_{\alpha \sigma}(\varepsilon)=\Gamma_{\alpha \sigma}(\varepsilon+\sigma \omega / 2)$.

The spin-resolved particle current flowing through lead $\alpha$ into the sample region, defined by $\left\langle J_{\alpha \sigma}(t)\right\rangle=-\left\langle d N_{\alpha \sigma} / d t\right\rangle$ with the number operator $N_{\alpha \sigma}$ for electrons in lead $\alpha$ with spin $\sigma$, is represented as

$$
\begin{aligned}
\left\langle J_{\alpha \sigma}(t)\right\rangle= & 2 \operatorname{Re} \int_{-\infty}^{\infty} d t^{\prime} \operatorname{Tr}\left[G_{\sigma \sigma}^{R}\left(t, t^{\prime}\right) \Sigma_{\alpha \sigma}^{<}\left(t^{\prime}-t\right)\right. \\
& \left.+G_{\sigma \sigma}^{<}\left(t, t^{\prime}\right) \Sigma_{\alpha \sigma}^{A}\left(t^{\prime}-t\right)\right],
\end{aligned}
$$

in terms of the nonequilibrium Green's functions, where $\Sigma_{\alpha \sigma}^{A}(t)=\left[\Sigma_{\alpha \sigma}^{R}(-t)\right]^{\dagger}$. This expression is reduced into an extended Landauer-Büttiker formula as follows: ${ }^{15}$

$$
\begin{aligned}
\left\langle J_{\alpha \sigma}(t)\right\rangle= & \frac{1}{2 \pi} \sum_{\beta} \int_{-\infty}^{\infty} d \varepsilon\left[T_{\beta \alpha \sigma}(\varepsilon) f_{\alpha \sigma}(\varepsilon)-T_{\alpha \beta \sigma}(\varepsilon) f_{\beta \sigma}(\varepsilon)\right] \\
& +\frac{1}{2 \pi} \sum_{\beta} \int_{-\infty}^{\infty} d \varepsilon\left[S_{\beta \alpha \sigma}(\varepsilon) f_{\alpha \sigma}(\varepsilon)\right. \\
& \left.-S_{\alpha \beta,-\sigma}(\varepsilon) f_{\beta,-\sigma}(\varepsilon)\right]
\end{aligned}
$$

where two types of transmission coefficients are defined as

$$
\begin{aligned}
& T_{\alpha \beta \sigma}(\varepsilon)=\operatorname{Tr}\left[\bar{\Gamma}_{\alpha \sigma}(\varepsilon) \tilde{G}_{\sigma}^{R}(\varepsilon) \bar{\Gamma}_{\beta \sigma}(\varepsilon) \tilde{G}_{\sigma}^{A}(\varepsilon)\right], \\
& S_{\alpha \beta \sigma}(\varepsilon)=\operatorname{Tr}\left[\widetilde{\Gamma}_{\alpha \sigma}(\varepsilon) \tilde{G}_{\sigma}^{R}(\varepsilon) \bar{\Gamma}_{\beta \sigma}(\varepsilon) \tilde{G}_{\sigma}^{A}(\varepsilon)\right],
\end{aligned}
$$

with $\widetilde{\Gamma}_{\alpha \sigma}(\varepsilon)=\widetilde{\Omega} \bar{G}_{-\sigma}^{R}(\varepsilon) \bar{\Gamma}_{\alpha,-\sigma}(\varepsilon) \bar{G}_{-\sigma}^{A}(\varepsilon) \widetilde{\Omega}$. Physically, $T_{\alpha \beta \sigma}$ $\left(S_{\alpha \beta \sigma}\right)$ describes the electron transportability from spin- $\sigma$ channel in lead $\beta$ to spin- $\sigma(-\sigma)$ channel in lead $\alpha$. Notice that $\left\langle J_{\alpha \sigma}(t)\right\rangle$ is independent of time even in the presence of the rotating field. It is easily found that the outward flow of the charge current $J_{\text {charge }}$ and that of the spin current $J_{\text {spin }}$ are expressed as

$$
\begin{gathered}
J_{\text {charge }}=-\sum_{\alpha, \sigma}\left\langle J_{\alpha \sigma}\right\rangle=0, \\
J_{\text {spin }}=-\frac{1}{2} \sum_{\alpha, \sigma} \sigma\left\langle J_{\alpha \sigma}\right\rangle=\frac{1}{2 \pi} \sum_{\alpha, \beta} \int_{-\infty}^{\infty} d \varepsilon\left[S_{\alpha \beta \downarrow}(\varepsilon) f_{\beta \downarrow}(\varepsilon)\right. \\
\left.-S_{\alpha \beta \uparrow}(\varepsilon) f_{\beta \uparrow}(\varepsilon)\right] .
\end{gathered}
$$

Obviously, Eq. (16) originates from the charge conservation and is explained in terms of the charge-current continuity [Eq. (1)], in combination with Gauss's divergence theorem. Similarly, Eq. (17) can be drawn from the spin-current continuity [Eq. (2)].

By definition, it is straightforward to show that

$$
\left\langle\rho_{\text {spin }}(\mathbf{r}, t)\right\rangle=-\frac{i}{2 a^{d}} \sum_{\sigma} \sigma\left[G_{\sigma \sigma}^{<}(t, t)\right]_{\mathbf{r r}},
$$

$$
\left\langle\mathbf{j}_{\text {spin }}(\mathbf{r}, t)\right\rangle=-\frac{t_{0}}{2 a^{d}} \sum_{\sigma} \sum_{ \pm, \mathbf{a}} \sigma\left( \pm \mathbf{a} \operatorname{Re}\left[G_{\sigma \sigma}^{<}(t, t)\right]_{\mathbf{r} \pm \mathbf{a}, \mathbf{r}}\right),
$$

$$
\left\langle g_{\text {spin }}(\mathbf{r}, t)\right\rangle=\frac{\widetilde{\omega}}{a^{d}} \operatorname{Re}\left[G_{\uparrow \downarrow}^{<}(t, t)\right]_{\mathrm{rr}} \exp (i \omega t) .
$$

All of these local quantities are time independent, because the equal-time correlation function $G_{\sigma \sigma^{\prime}}^{<}(t, t)$ involved in Eq. (18) is given by $G_{\sigma \sigma}^{<}(t, t)=(2 \pi)^{-1} \int_{-\infty}^{\infty} d \varepsilon R_{\sigma \sigma}^{<}(\varepsilon)$ or $G_{\sigma,-\sigma}^{<}(t, t)$ $=(2 \pi)^{-1} \int_{-\infty}^{\infty} d \varepsilon R_{\sigma,-\sigma}^{<}(\varepsilon) \exp (-i \sigma \omega t)$. Hence, the local continuity, $\left\langle\nabla \cdot \mathbf{j}_{\text {spin }}(\mathbf{r})\right\rangle=\left\langle g_{\text {spin }}(\mathbf{r})\right\rangle$, provides a conservation law for spin flux, which can be expressed as

$$
J_{\text {spin }}=\oint_{S}\left\langle\mathbf{j}_{\text {spin }}(\mathbf{r})\right\rangle \cdot \mathbf{n} d S=\int_{V}\left\langle g_{\text {spin }}(\mathbf{r})\right\rangle d V=G_{\text {spin }},
$$

for $d=3$, where the surface $S$ encloses the sample of volume $V$, and $\mathbf{n}$ is the outward-directed unit vector normal to $S$. The spin generation rate integrated over the sample region, formulated as $G_{\text {spin }}=\pi^{-1} \operatorname{Re} \int_{-\infty}^{\infty} d \varepsilon \operatorname{Tr}\left[\widetilde{\Omega} R_{\uparrow \downarrow}^{<}(\varepsilon)\right]$, leads to exactly the same expression as Eq. (17) for $J_{\text {spin. }}$

The expression for $J_{\text {spin }}$ or equivalently $G_{\text {spin }}$ is further simplified by considering the identical electrochemical potential for all leads. In this situation, a pure spin current is pumped without any charge current. A sum rule that $\Sigma_{\alpha, \beta} S_{\alpha \beta \uparrow}(\varepsilon)=\Sigma_{\alpha, \beta} S_{\alpha \beta \downarrow}(\varepsilon)$ for the spin-flip transport yields

$$
J_{\text {spin }}=\frac{1}{2 \pi} \int_{-\infty}^{\infty} d \varepsilon\left[f_{\downarrow}(\varepsilon)-f_{\uparrow}(\varepsilon)\right] \operatorname{Tr}\left[\widetilde{\Gamma}_{\uparrow}(\varepsilon) \widetilde{G}_{\uparrow}^{R}(\varepsilon) \bar{\Gamma}_{\uparrow}(\varepsilon) \widetilde{G}_{\uparrow}^{A}(\varepsilon)\right],
$$

where $\quad \bar{\Gamma}_{\sigma}(\varepsilon)=\Sigma_{\alpha} \bar{\Gamma}_{\alpha \sigma}(\varepsilon)=-2 \operatorname{Im} \bar{\Sigma}_{\sigma}^{R}(\varepsilon) \quad$ and $\quad \tilde{\Gamma}_{\sigma}(\varepsilon)$ $=\Sigma_{\alpha} \widetilde{\Gamma}_{\alpha \sigma}(\varepsilon)=-2 \operatorname{Im} \widetilde{\Sigma}_{\sigma}^{R}(\varepsilon)$. Therefore, the pumped spin per one cycle of field rotation, $S_{\text {pump }}=(2 \pi / \omega) J_{\text {spin }}$, can be simply written as

$$
S_{\text {pump }}=\operatorname{Tr}\left[\widetilde{\Gamma}_{\uparrow}(E) \widetilde{G}_{\uparrow}^{R}(E) \bar{\Gamma}_{\uparrow}(E) \widetilde{G}_{\uparrow}^{A}(E)\right],
$$

at zero temperature and in the adiabatic regime [in this regime, $\omega$ is sufficiently small such that $f_{\downarrow}(\varepsilon)-f_{\uparrow}(\varepsilon)=\omega \delta(\varepsilon$ $-E)$ ], where $E$ is the equilibrium Fermi energy. The theoretical formulation described in this paper is quite general. It is easily shown that the present theory reproduces the previous result obtained for a quantum-dot spin pump in the so-called wideband limit assuming that the retarded self-energy due to the lead does not depend on energy. ${ }^{11}$

It may be interesting to compare the theoretical result based on the nonequilibrium Green's-function formalism with that derived from the Kubo formula for an infinitely large homogeneous system. ${ }^{16}$ For this purpose, we consider the Hamiltonian consisting of $H_{0}+\widetilde{H}(t)$, where $H_{0}$ $=\Sigma_{\mathbf{k}, \sigma} \varepsilon_{\mathbf{k} \sigma} c_{\mathbf{k} \sigma}^{\dagger} c_{\mathbf{k} \sigma}$ incorporates the Zeeman interaction induced by the static field into the particle energy $\varepsilon_{\mathbf{k} \sigma}=\varepsilon_{\mathbf{k}}+\sigma \bar{\omega} / 2$, and $\tilde{H}(t)=(\widetilde{\omega} / 2) \Sigma_{ \pm} S_{ \pm} \exp (\mp i \omega t)$ describes the interaction arising from the rotating field. The fermion operators, $c_{\mathbf{k} \sigma}$ and $c_{\mathbf{k} \sigma}^{\dagger}$, are defined in momentum space, and the spin- 
raising and -lowering operators, $S_{+}=\Sigma_{\mathbf{k}} c_{\mathbf{k} \uparrow}^{\dagger} c_{\mathbf{k} \downarrow}$ and $S_{-}$ $=\Sigma_{\mathbf{k}} c_{\mathbf{k} \downarrow}^{\dagger} c_{\mathbf{k} \uparrow}$, are written in a second-quantization fashion. Treating $\widetilde{H}(t)$ as a perturbation, the Kubo formula evaluates the total spin generation rate to be

$$
\begin{aligned}
G_{\text {spin }} & =\frac{1}{2} \widetilde{\omega}^{2} \operatorname{Im} \chi_{-+}(\omega) \\
& =\frac{\pi}{2} \widetilde{\omega}^{2} \delta(\omega-\bar{\omega}) \int_{-\infty}^{\infty} d \varepsilon\left[f_{\downarrow}(\varepsilon)-f_{\uparrow}(\varepsilon)\right] N(\varepsilon),
\end{aligned}
$$

where $\chi_{-+}(\omega)$ is the Fourier transform of the retarded correlation function of spin operators $\chi_{-+}(t)=i\left\langle\left[S_{-}(t), S_{+}(0)\right]\right\rangle \theta(t)$, and $N(\varepsilon)=\Sigma_{\mathbf{k}} \delta\left(\varepsilon-\varepsilon_{\mathbf{k}}\right)$ is the density of states per spin. In this expression, the quadratic response $G_{\text {spin }} \propto \widetilde{\omega}^{2}$ is obvious for a weak rotating field. The delta function $\delta(\omega-\bar{\omega})$ accounts for the paramagnetic resonance at $\omega=\bar{\omega}$ involved in the spin generation mechanism. On the other hand, Eq. (20) obtained for a finite-sized sample without suffering voltage bias is used to derive a perturbative result which is valid for a sufficiently small $\widetilde{\omega}$,

$$
J_{\text {spin }}=\frac{1}{8 \pi} \widetilde{\omega}^{2} \int_{-\infty}^{\infty} d \varepsilon\left[f_{\downarrow}(\varepsilon)-f_{\uparrow}(\varepsilon)\right] \operatorname{Tr}\left[\bar{A}_{\uparrow}(\varepsilon) \bar{A}_{\downarrow}(\varepsilon)\right],
$$

where $\bar{A}_{\sigma}(\varepsilon)=\bar{G}_{\sigma}^{R}(\varepsilon) \bar{\Gamma}_{\sigma}(\varepsilon) \bar{G}_{\sigma}^{A}(\varepsilon)$ is the spectral function. If the sample size is infinitely large, $\bar{A}_{\sigma}(\varepsilon)$ approximates to $2 \pi \delta\left(\varepsilon+\sigma \omega / 2-H_{\sigma}\right)$. Then, Eq. (23) coincides with Eq. (22).

\section{NUMERICAL CALCULATION AND DISCUSSION}

The numerical calculations based on the nonequilibrium Green's-function formalism have been performed for quantitatively studying the spin generation and the spin transport in $d=1$ and 2 finite-sized systems. The $d=1$ system has a sample region of length $L$ connected to two semi-infinite leads at both ends, which are also one dimensional. The $d$ $=2$ system is the four-terminal bridge consisting of a central square sample of size $L \times L$ and four leads of the same width $L$ attached symmetrically on all four sides of the sample. These configurations were chosen for no particular reason. In principle, the spin pump will work in any structure with proper spin-current probes. The calculation assumes the same chemical potential for all leads and zero temperature. In the following, the hopping energy $t_{0}$ is taken as the energy unit $\left(t_{0}=1\right)$, and the lattice spacing $a$ as the length unit ( $a$ $=1$ ). The parameters are normally $\bar{\omega}=0, \widetilde{\omega}=2 \times 10^{-4}, E=1$, and $\omega \rightarrow 0$ (which corresponds to the adiabatic limit) if there is no reference to their values.

Figure 1 summarizes the pumped spin $S_{\text {pump }}$ per one cycle of field rotation calculated as a function of the Fermi energy $E$ for the $d=1$ and 2 systems with different sample sizes $L$. The variation of $S_{\text {pump }}$ with $E$ is symmetric about the center of the energy band and reflects its density of states as implied from Eq. (22) for an infinite system. A small oscillation for $d=2$ is due to the subband formation in the finite-sized sample. The spin current pumped from the sample increases with its size, indicating the spin generation over a whole region of the sample. In order to gain a microscopic insight
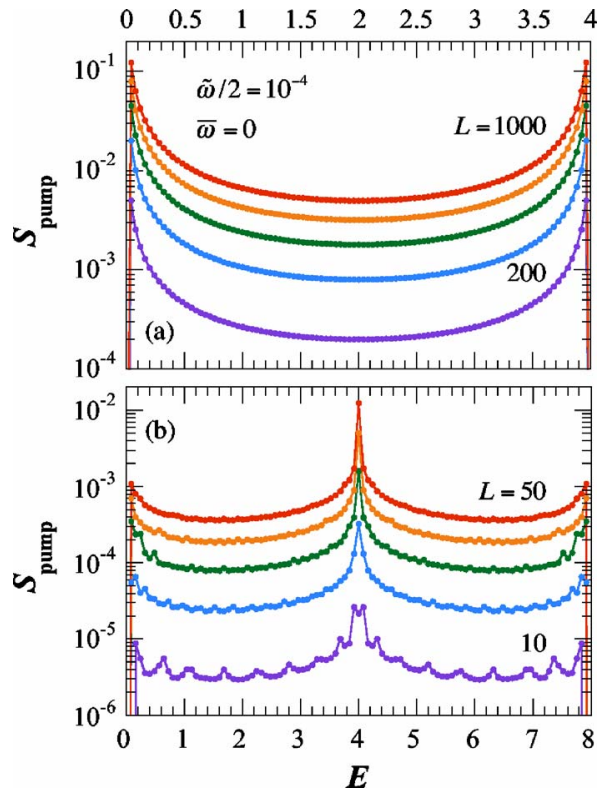

FIG. 1. (Color online) Pumped spin as a function of Fermi energy calculated (a) for $d=1$ systems with $L=200,400,600,800$, and 1000 and (b) for $d=2$ systems with $L=10,20,30,40$, and 50 . The parameters used in the calculation are indicated in the figure.

into the spin-pumping mechanism, it is useful to visualize the spatial profiles of local quantities, such as the spin density, the spin-current density, and the spin generation rate. Figure 2 displays the results of calculation for the $d=2$ system with $L=80$ (for the sake of visibility, $L=40$ for spin current). In this calculation, it is assumed that the region of a central $40 \times 40(20 \times 20$ for spin current $)$ square is partially pumped with the rotating field for observing the spin injection into the outer region where the pumping field is absent. The spin generation rate is almost uniformly distributed over the pumped region, producing a spin current that flows radially. The local spin current is amplified with distance from the center and reaches a maximum around the edges of the pumped region. These observations are easily interpreted in view of Gauss's divergence theorem. The spin current pumped from the inner region flows continuously into the outer region and is finally absorbed into the leads attached on four sides of the sample. It is also confirmed that the divergent spin current extends the spin population toward the leads.

Figure 3 displays the sample-size dependencies of the pumped spin $S_{\text {pump }}$ calculated for $d=1$ and 2 . It is clearly found that $S_{\text {pump }}$ follows a simple power-law dependence on $L$, except for a small fluctuation for $d=2$ due to the subband formation. Figure 4 illustrates how $S_{\text {pump }}$ varies with the frequency difference $\delta \omega=\omega-\bar{\omega}$ when the rotating field is off resonance. The upper figure shows the $\bar{\omega}$ dependencies calculated in the limit $\omega \rightarrow 0$, while the lower figure shows the $\omega$ dependencies at $\bar{\omega}=0$ for the $d=2$ systems having various sample sizes. The pumped spin is kept constant over a moderate range of detuning $|\delta \omega|$ from resonance, whereas it decreases rapidly when $|\delta \omega|$ becomes extremely large. The observations for $d=1$ are similar (not shown). A semiclassical consideration is useful for interpreting these observations. 

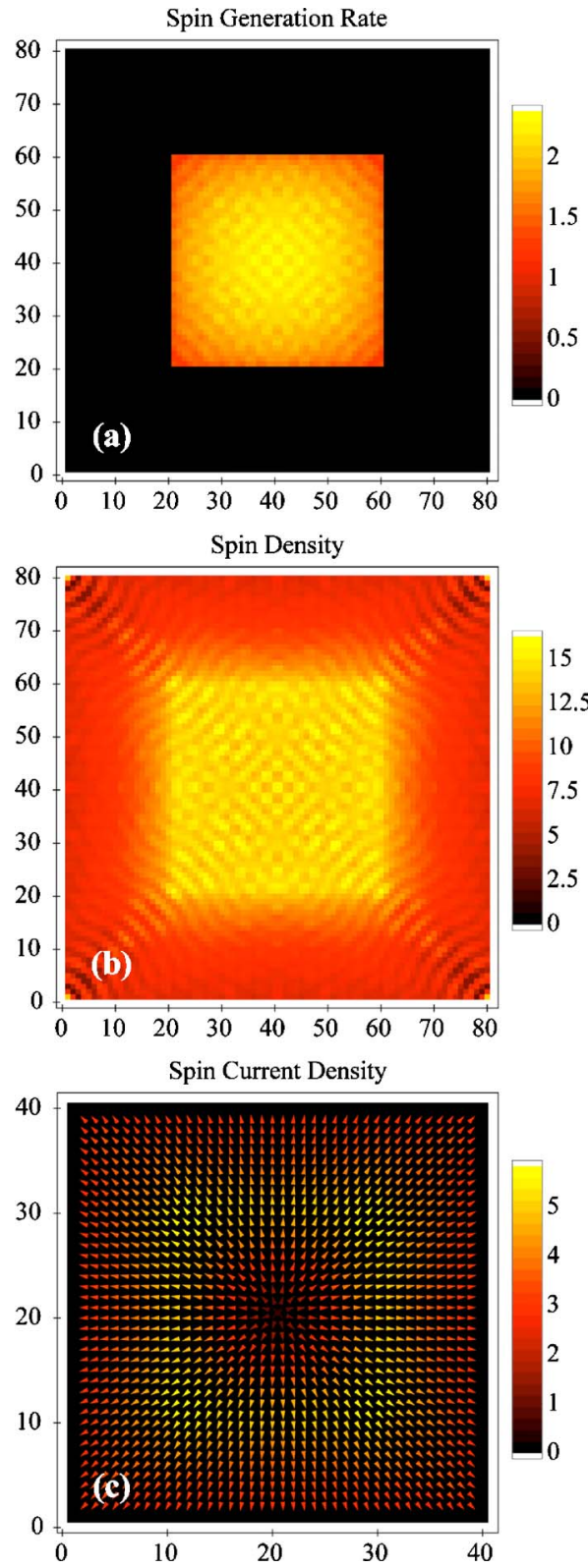

FIG. 2. (Color online) (a) Spin generation rate, (b) spin-density, and (c) spin-current density normalized by a factor $\omega(\widetilde{\omega} / 2)^{2}$. The sample size is $L=80$ for (a) and (b), while $L=40$ for (c). The calculation assumes that a central $L / 2 \times L / 2$ square is adiabatically pumped with the rotating field. The parameters used in the calculation are $\bar{\omega}=0, \widetilde{\omega}=2 \times 10^{-4}$, and $E=1$.

For a finite-sized sample connected to a lead into which an electron can escape out, the retarded self-energy may be treated approximately as $\Sigma^{R} \cong-i / 2 \tau$, where $\tau$ represents the lifetime or the average time an electron remains in the sample region. ${ }^{15}$ In accordance with Eq. (23), this approximation allows us to write a low-frequency response as

$$
J_{\text {spin }} \cong \frac{\widetilde{\omega}^{2}}{2} \frac{\omega \tau_{F}}{1+\left(\delta \omega \tau_{F}\right)^{2}} N(E),
$$

assuming that $|\omega| / 2 \ll E$ and the lifetime $\tau_{F}=L / 2 v_{F}$ (where $v_{F}$ is the Fermi velocity) is large enough but finite. This

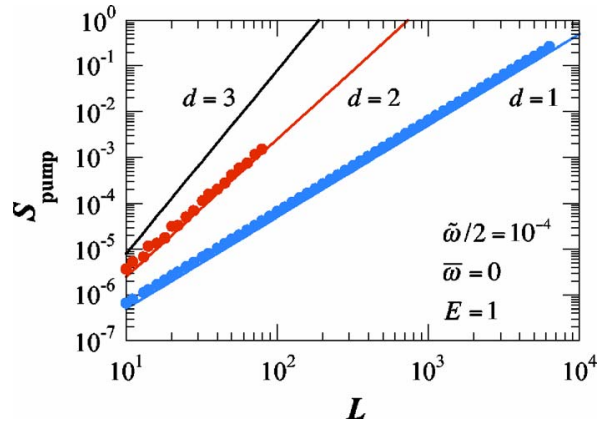

FIG. 3. (Color online) Pumped spin as a function of sample size. The parameters used in the calculation are indicated in the figure. The solid line shows the theoretical plot according to Eq. (25).

simple formula is valid beyond the adiabatic regime where $J_{\text {spin }}$ is linear in $\omega$, giving a semiquantitative interpretation for the resonance linewidth. For the free-particle dispersion, $\varepsilon_{\mathbf{k}}=k^{2} / 2 m$, Eq. (24) predicts

$$
S_{\text {pump }} \cong \frac{\widetilde{\omega}^{2}}{4} \times \begin{cases}(m / E) L^{2}, & d=1, \\ \left(m / v_{F}\right) L^{3}, & d=2, \\ \left(m^{2} / \pi\right) L^{4}, & d=3,\end{cases}
$$

at resonance $\delta \omega=0$. In Fig. 3, the analytical results following Eq. (25) are shown by solid lines, which yield a satisfactory explanation to the numerical results. It has been confirmed in the calculation that the agreement becomes better for lower Fermi energies.

So far, we have addressed the numerical results obtained for a relatively weak rotating field $\left(\tilde{\omega}=2 \times 10^{-4}\right)$. We discuss here how $S_{\text {pump }}$ depends on $\widetilde{\omega}$. Figure 5 displays the typical

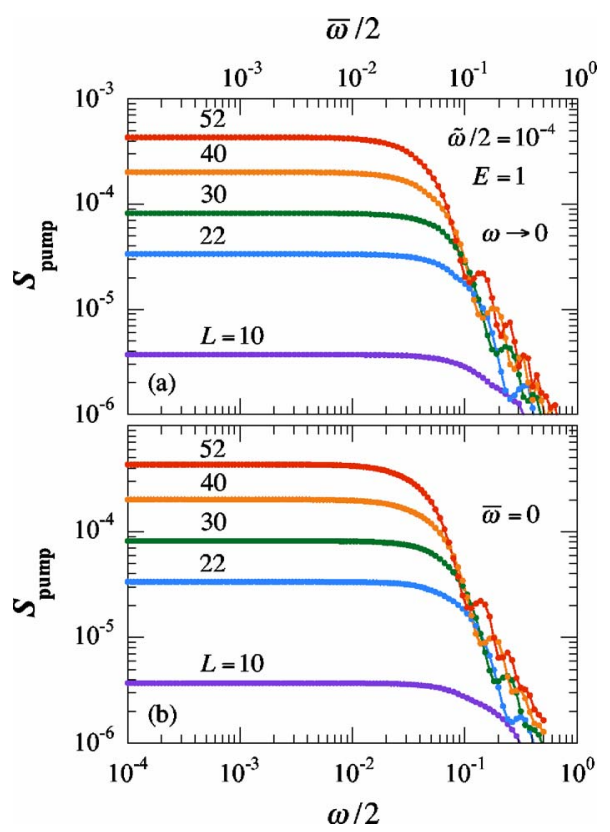

FIG. 4. (Color online) Pumped spin versus (a) Larmor frequency of static field and (b) rotation frequency calculated for $d$ $=2$ systems with $L=10,22,30,40$, and 52 . The parameters used in the calculation are indicated in the figure. 


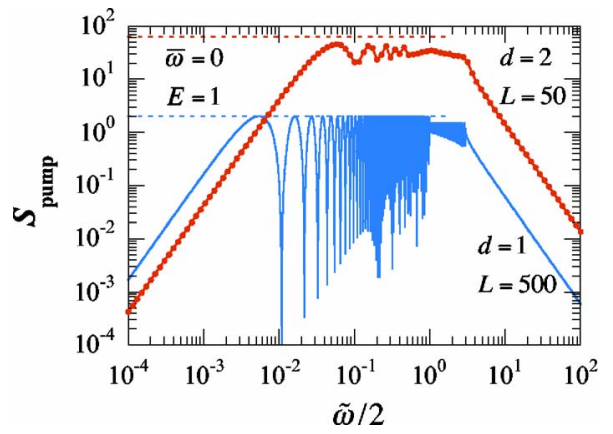

FIG. 5. (Color online) Pumped spin as a function of Larmor frequency of rotating field calculated for $d=1$ and 2 systems. The parameters used in the calculation are indicated in the figure. The dashed line represents the upper limit $S_{\max }$.

results calculated for $d=1$ and 2. At first glance, we find three distinctive regions. When $\widetilde{\omega}$ is small, $S_{\text {pump }}$ has a quadratic dependence on $\widetilde{\omega}$. This behavior is quantitatively explained by Eq. (23) derived as the lowest-order approximation. When $\widetilde{\omega}$ becomes extremely large, $S_{\text {pump }}$ decreases monotonically with $\widetilde{\omega}$. Such a trend may arise from an excessive discontinuity at the boundary between the sample exposed to the rotating field and the field-free lead, in terms of particle energy as well as density of states. This interpretation is corroborated from the observation that all the transmission coefficients defined by Eqs. (14) and (15) tend to vanish in this regime (not shown). The remaining problem is the medium- $\widetilde{\omega}$ region, where some upper bound exists and $S_{\text {pump }}$ oscillates with $\widetilde{\omega}$ below it. The saturation of $S_{\text {pump }}$ may be ascribed to a limited number of channels (or transverse modes for $d=2$ ) in the lead that transports spin. The transmission coefficients satisfy the relation that $M_{\alpha \sigma}-R_{\alpha \sigma}$ $=\Sigma_{\beta \neq \alpha} T_{\beta \alpha \sigma}+\Sigma_{\beta} S_{\beta \alpha \sigma}$, where $M_{\alpha \sigma}$ is the number of spin- $\sigma$ channels in lead $\alpha$, and $R_{\alpha \sigma}$ is the spin-conserving reflection coefficient. Its direct consequence is that $S_{\text {pump }} \leqslant S_{\max }$ $\equiv(1 / 2) \Sigma_{\alpha, \sigma} M_{\alpha \sigma}$. For $d=1$, there are two channels per lead for spin- $\uparrow$ and spin- $\downarrow$ states so that $S_{\max }=2$ for two leads, which is consistent with the observation. The saturation observed for the four-terminal $d=2$ system of $L=50$ can also be explained by the number of channels in leads, for which $S_{\max }=64$ at $E=1$. The number of transport channels per spin is roughly evaluated to be $M=1$ for $d=1, M=k_{F} L / \pi$ for $d$ $=2$, and $M=k_{F}^{2} L^{2} / 4 \pi$ for $d=3$ (where $k_{F}$ is the Fermi momentum). Comparing these expressions to Eq. (25) leads us to find that $S_{\text {pump }}$ saturates when $\widetilde{\omega} \tau_{F} \geqslant 1$, irrespective of dimensionality.

The disorder effect may be an important issue when considering the observability of spin pumping from realistic systems, which are not perfectly clean but usually contain a number of impurities. In this study, the static disorder caused by nonmagnetic impurities is taken into account by introducing a random on-site potential $V(\mathbf{r})$ uniformly distributed in the range $-W / 2 \leqslant V(\mathbf{r}) \leqslant W / 2$. The results of calculation for $d=2$ are shown in Fig. 6, where we clearly find that the spin pumping is not only robust against a weak disorder but also largely enhanced by a strong disorder. The enhancement occurs in the diffusive transport regime in which the elastic mean free path $\ell=v_{F} \tau$ (where $\tau$ is the momentum relaxation

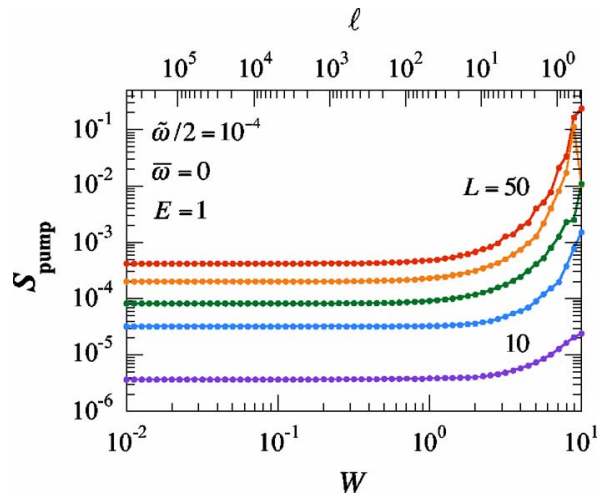

FIG. 6. (Color online) Pumped spin as a function of disorder strength calculated for $d=2$ systems with $L=10,20,30,40$, and 50 . The parameters used in the calculation are indicated in the figure. In the calculation, disorder average is performed over 1000 random configurations. The upper horizontal axis is scaled with the elastic mean free path estimated from the lowest-order Born approximation as a reference.

time) is shorter than the sample size $L$. In a classical treatment, a Brownian particle with a diffusion coefficient $D$ spends the time $L^{2} / D$ passing through a sample of length $L$. In view of this, the transit time, during which an electron remains in the sample region and is exposed to the rotating field, is elongated by a factor $d L / \ell$ for the diffusive transport with $D=\ell^{2} / d \tau$, giving a greater probability of spin flip. Although this explanation is merely qualitative, it seems to reasonably describe the disorder effect on spin pumping. A more quantitative analysis can be performed by employing the diagrammatic perturbation theory. In analogy with the weaklocalization correction to electrical conductivity, ${ }^{19}$ we consider here the maximally crossed diagram contributing to the spin-spin correlation function $\chi_{-+}(\omega)$, which consists of the particle-hole propagator with antiparallel spins subject to scattering due to random impurities, as shown in Fig. 7. The spin-spin correlation function is systematically calculated from the summation of the geometric series of maximally crossed diagrams. The imaginary part $\operatorname{Im} \chi_{-+}(\omega)$, which is of particular importance since it is directly related to the spin generation rate $G_{\text {spin }}=\left(\widetilde{\omega}^{2} / 2\right) \operatorname{Im} \chi_{-+}(\omega)$, is found to be $\operatorname{Im} \chi_{-+}=N(E) \omega \tau+\left(d \omega / \pi v_{F}^{2}\right) \Sigma_{\mathrm{q}} q^{-2}$ for $\omega=\bar{\omega}$. It is noticed that the second term is divergent for $q \cong 0$, describing a singular backscattering $(\mathbf{k} \rightarrow-\mathbf{k})$ due to the random potential, which is crucial for the localization caused by quantum interference. The lower momentum cutoff for a system of size $L$ is $q \approx 1 / L$, the upper cutoff being $q \approx 1 / \ell$. Thus, we obtain

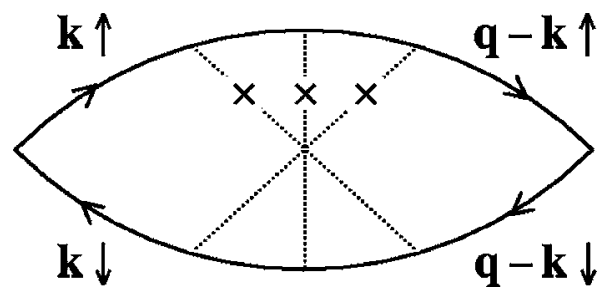

FIG. 7. Maximally crossed diagram for particle-hole propagator with antiparallel spins. The dashed line with a cross denotes impurity scattering. 


$$
\begin{aligned}
\operatorname{Im} \chi_{-+}= & N(E) \omega \tau+\frac{\omega}{\pi^{2} v_{F}^{2}} \\
& \times\left\{\begin{array}{cc}
L(L-\ell), & d=1, \\
L^{2} \ln (L / \ell), & d=2, \\
(3 / 2 \pi) L^{3}(1 / \ell-1 / L), & d=3,
\end{array}\right.
\end{aligned}
$$

for $d$-dimensional finite-sized systems. The second term is analogous to the quantum correction for conductivity but has an opposite sign and explains that the weak localization assists the spin pumping.

Finally, we consider the magnitude of spin-current pumped from realistic systems. The spin-pumping system should be smaller than the spin-diffusion length $L_{\text {spin, which }}$ determines the spatial scale over which the spin polarization is preserved, and is mainly ruled by the momentum relaxation time $\tau$ as well as the longitudinal spin-relaxation time $\tau_{\text {spin. }}$ In a typical metal such as $\mathrm{Al}, \tau_{\text {spin }}$ exceeds $1 \mathrm{~ns}$ at low temperatures while $\tau$ is on the order of $10 \mathrm{fs}^{20}$ The Fermi velocity $v_{F} \cong 2 \times 10^{8} \mathrm{~cm} / \mathrm{s}$ for Al gives an estimate for the spin-diffusion length at $L_{\text {spin }} \cong 3 \mu \mathrm{m}$, which is very long relative to the elastic mean free path $\ell \cong 20 \mathrm{~nm}$. A longer spin-diffusion length may be expected for semiconductors than for metals. As for III-V semiconductor compounds, there is a wealth of useful data on $\tau_{\text {spin. }}$. Compared to holes, electrons in III-V systems usually have a longer $\tau_{\text {spin }}$ owing to a relatively weak spin-orbit coupling and are more suitable for spin pumping. Typical values of $\tau_{\text {spin }}$ measured for electrons range from $10 \mathrm{ps}$ to $100 \mathrm{~ns} .{ }^{20} \mathrm{Si}$-based heterostructures are also interesting candidates for spin pumping. The spin relaxation of a two-dimensional electron gas has been investigated by electron-spin resonance in modulation-doped $\mathrm{Si} / \mathrm{SiGe}$ quantum wells. High-mobility samples with electron density of $3 \times 10^{11} \mathrm{~cm}^{-2}$ exhibit $\tau_{\text {spin }}$ of up to $30 \mu \mathrm{s}$ at low temperatures, ${ }^{21-23}$ for which the spin-diffusion length amounts to $L_{\text {spin }} \cong 700 \mu \mathrm{m}$, assuming $\tau=10$ ps. Based on these considerations, we suppose here a two-dimensional system of size $L=500 \mu \mathrm{m}$. The spin-pumping experiment may be made using a standard $X$-band $(9 \mathrm{GHz})$ electronspin-resonance spectrometer, in which the magnetic component of the microwave field can be varied to as large as $\widetilde{B}$ $=100 \mu \mathrm{T}$. In this case, Eq. (25) evaluates the pumped spin per one cycle of field rotation to be $S_{\text {pump }} \cong 300$ at resonance (which is much smaller than the number of transport channels). The resulting spin current $J_{\text {spin }} \cong 3 \times 10^{12} \mathrm{~s}^{-1}$ is quite large, which is equivalent to a spin-polarized charge current of $1 \mu \mathrm{A}$. It should be noted that this estimation is valid only for the ballistic transport. In the diffusive transport regime, which is practical for the present consideration since $\ell \ll L$ $\leqslant L_{\text {spin }}$, there is a possibility that the spin pumping is enhanced further.

\section{CONCLUSIONS}

In conclusion, we have studied the spin pumping due to a rotating magnetic field applied to finite-sized electron systems in the ballistic and diffusive transport regimes, on the basis of the nonequilibrium Green's-function formalism. The rotating field generates a time-independent dc spin current, which is not accompanied by any charge current when the electrochemical potentials in all leads attached to the system are identical. The physics of spin pumping is easily understood in terms of the spin-current continuity equation with the source term attributable to the spin torque due to the rotating field, leading to the identity $J_{\text {spin }}=G_{\text {spin }}$. The magnitude of pumped spin per one cycle of field rotation depends largely on the dimensionality, the system size, the intensity of the rotating field, the detuning from resonance, and the static disorder. In the ballistic transport regime, the pumped spin at resonance is proportional to $\widetilde{\omega}^{2} L^{d+1}$, when the system contacts the ideal leads containing enough channels for spin transport, and the pumping field is perturbatively weak. In the diffusive transport regime, the spin pumping is dramatically enhanced with increasing static disorder. The mechanism of enhancement is explained basically in view of the weak localization.
${ }^{1}$ M. Johnson and R. H. Silsbee, Phys. Rev. Lett. 55, 1790 (1985).

${ }^{2}$ F. J. Jedema, A. T. Filip, and B. J. van Wees, Nature (London) 410, 345 (2001).

${ }^{3}$ R. Fiederling, M. Keim, G. Reuscher, W. Ossau, G. Schmidt, A. Waag, and L. W. Molenkamp, Nature (London) 402, 787 (1999).

${ }^{4}$ Y. Ohno, D. K. Young, B. Beschoten, F. Matsukura, H. Ohno, and D. D. Awschalom, Nature (London) 402, 790 (1999).

${ }^{5}$ A. Brataas, Y. Tserkovnyak, G. E. W. Bauer, and B. I. Halperin, Phys. Rev. B 66, 060404(R) (2002).

${ }^{6}$ E. R. Mucciolo, C. Chamon, and C. M. Marcus, Phys. Rev. Lett. 89, 146802 (2002).

${ }^{7}$ Q.-F. Sun, H. Guo, and J. Wang, Phys. Rev. Lett. 90, 258301 (2003).

${ }^{8}$ E. Cota, R. Aguado, and G. Platero, Phys. Rev. Lett. 94, 107202 (2005)

${ }^{9}$ S. Murakami, N. Nagaosa, and S.-C. Zhang, Phys. Rev. B 69,
235206 (2004).

${ }^{10}$ J. Sinova, D. Culcer, Q. Niu, N. A. Sinitsyn, T. Jungwirth, and A. H. MacDonald, Phys. Rev. Lett. 92, 126603 (2004).

${ }^{11}$ B. Wang, J. Wang, and H. Guo, Phys. Rev. B 67, 092408 (2003).

${ }^{12}$ P. Zhang, Q.-K. Xue, and X. C. Xie, Phys. Rev. Lett. 91, 196602 (2003).

${ }^{13}$ B. Dong, H. L. Cui, and X. L. Lei, Phys. Rev. Lett. 94, 066601 (2005).

${ }^{14}$ A.-P. Jauho, N. S. Wingreen, and Y. Meir, Phys. Rev. B 50, 5528 (1994).

${ }^{15}$ S. Datta, Electronic Transport in Mesoscopic Systems (Cambridge University Press, Cambridge, 1995).

${ }^{16}$ G. D. Mahan, Many-Particle Physics (Kluwer Academic, Dordrecht/Plenum, New York, 2000).

${ }^{17}$ A. Dhar and D. Sen, Phys. Rev. B 73, 085119 (2006).

${ }^{18}$ It has been confirmed in the numerical calculation that the orbital effects give no significant effect on $S_{\text {pump }}$ at resonance except for 
an appearance of small peaks due to the Landau-level formation.

${ }^{19}$ P. A. Lee and T. V. Ramakrishnan, Rev. Mod. Phys. 57, 287 (1985)

${ }^{20}$ I. Zutic, J. Fabian, and S. D. Sarma, Rev. Mod. Phys. 76, 323 (2004).
${ }^{21}$ C. F. O. Graeff, M. S. Brandt, M. Stutzmann, M. Holzmann, G. Abstreiter, and F. Schäffler, Phys. Rev. B 59, 13242 (1999).

${ }^{22}$ N. Sandersfeld, W. Jantsch, Z. Wilamowski, and F. Schäffler, Thin Solid Films 369, 312 (2000).

${ }^{23}$ Z. Wilamowski and W. Jantsch, cond-mat/0112466 (unpublished). 